TO APPEAR IN APJ

Preprint typeset using LTTEX style emulateapj v. 03/07/07

\title{
CONSTRAINING PULSAR MAGNETOSPHERE GEOMETRY WITH $\gamma$-RAY LIGHT CURVES
}

\author{
Roger W. Romani ${ }^{1}$ AND KYLE P. WATters ${ }^{1}$ \\ To appear in ApJ
}

\begin{abstract}
We demonstrate a method for quantitatively comparing $\gamma$-ray pulsar light curves with magnetosphere beaming models. With the Fermi LAT providing many pulsar discoveries and high quality pulsar light curves for the brighter objects, such comparison allows greatly improved constraints on the emission zone geometry and the magnetospheric physics. Here we apply the method to Fermi LAT light curves of a set of bright pulsars known since EGRET or before. We test three approximate models for the magnetosphere structure and two popular schemes for the location of the emission zone, the Two Pole Caustic (TPC) model and the Outer Gap (OG) model. We find that OG models and relatively physical $B$ fields approximating force-free dipole magnetospheres are preferred at high statistical significance. An application to the full LAT pulsar sample will allow us to follow the emission zone's evolution with pulsar spindown.
\end{abstract}

Subject headings: gamma rays: stars - pulsars: general

\section{INTRODUCTION}

With the successful launch of Fermi, formerly GLAST, the number and quality of $\gamma$-ray light curves available for comparison with magnetospheric models has substantially improved (Abdo et al 2010). The quality and uniformity of the data make this a good time to down-select models for pulsed magnetospheric emission, by comparison with the observed curves. In the present paper we describe a method of attack for this process and apply it to the new improved light curves provided by Fermi for several well-known $\gamma$-ray pulsars.

The most popular pulsar models postulate regions in the magnetosphere where the force-free perfect $\operatorname{MHD}(\vec{E} \cdot \vec{B}=0)$ conditions break down, and in response to the uncanceled rotation-induced EMF $\vec{E} \approx-\vec{r} \times \vec{\Omega} \times \vec{B} / c$, the charges re-arrange so that non-negligible fields penetrate these lower density 'gap' zones, causing particle acceleration and radiation. Traditionally, one approximates the magnetosphere by a vacuum dipole field.

There are three locations commonly discussed for these magnetospheric gaps. The earliest pulsar models focused on acceleration at the foot points of the open field line zone, the so-called "polar caps". This emission should arise from within about a stellar radius (Daugherty \& Harding 1996), and should therefore suffer strong attenuation from one photon $(\gamma-\mathrm{B})$ pair creation. The corresponding hyper-exponential cutoffs are not seen in the LAT pulsar spectra, and so it has been concluded that the bulk of the pulsar emission must come from higher altitudes (Abdo et al 2010). The first high altitude model posits "Holloway" gaps above the null charge surface, extending toward the light cylinder at $r_{L C}=c / \Omega$ (the "outer gap" model, Cheng, Ho \& Ruderman 1986; Romani 1996). More recently, it has been argued that acceleration at the rims of the polar caps may extend to very high altitude (Muslimov and Harding 2004), effectively pushing the polar cap activity outward. In this 'slot gap'-type picture emission can occur over a large fraction of the boundary of the open zone. The geometrical realization of this model makes low altitude emission visible from one hemisphere and higher altitude $\left(r>r_{N C}\right)$ emission visible from the other. This is

\footnotetext{
Electronic address: rwr@astro.stanford.edu, kwatters@stanford.edu
}

${ }^{1}$ Department of Physics, Stanford University, Stanford, CA 94305 the "two pole caustic" (TPC) model (Dyks and Rudak 2003) which is thus intermediate between the polar cap (PC) and outer gap (OG) pictures.

In Watters et al. (2009), we created a library of light curves for these three emission locations in a dipole field and classified the phases of the dominant peaks. The results were presented as an 'atlas' of pulse properties which could be used to predict the pulse multiplicity and phase separation of a given model for a particular viewing geometry. While this is useful for an overview of the possible light curves and provides a convenient reference to read off the allowed angles for a given model, the complexity of the modeled beams meant that an appropriate phase separation could be found in several models for any one pulsar.

These computations implicitly assume static co-rotating charges only. Of course, the presence of radiating pair plasma in the magnetosphere requires some particle production, and so the vacuum plus co-rotating charge model will be approximate at best. Therefore an alternative attack is based on numerical solutions for magnetospheric charges, currents and fields, assuming that pair production is so robust that the force-free condition holds everywhere (Contopolous, Kazanas \& Fendt 1999; Spitkovsky 2006; Bai \& Spitkovsky 2009b). Such filled magnetospheres lack the acceleration fields required to produce powerful $\gamma$ ray beams, so the truth must lie in between, with some regions experiencing charge starvation and departing from force-free.

Here we wish to show how the actual light curves from the computations can be compared with the data. We demonstrate that the present data quality are sufficient to start comparing predictions between different assumed magnetic field structures and emission zone locations. In particular, while in many cases several models can produce plausible matches to the light curves for some angle, when we restrict our attention to the geometries demanded by external constraints, one can often strongly exclude certain models.

For our model set, we start by amending certain inadequacies in the vacuum computations pointed out by Bai \& Spitkovsky (2009a). This ends up making modest differences to the results in Watters et al (2009). We also introduce a simple model to illustrate the field structure perturbations resulting from open-zone currents. Comparing with 
the Fermi full band light curves, we find that lower altitude TPC-type models have great difficulty producing acceptable matches for many pulsars for all of these field geometries (at least for the assumptions made here) and that OG-type models are statistically strongly preferred. Further, in Fermi-quality data we find that the light curve matches can often be significantly improved by the magnetospheric current perturbations. In a following communication, we will apply such analysis to the full set of Fermi LAT pulsars, marry the light curve fitting code to a population synthesis model, and extract additional information on the pulsar beam structure and its evolution with age as the pulsar spins down.

\section{MODELING METHOD}

Our basic assumptions are that $i$ ) the stable, time-averaged pulse profile is caused by emission zones locked to a set of 'open' field lines, $i i$ ) these field structures are dominated by the dipole component of the field anchored in the star, since for young pulsars this emission occurs at many $\left.R_{*}, i i i\right)$ that a co-rotation charge density distributed throughout the bulk of the magnetosphere causes the plasma to follow the field lines and rotate with the star and $i v$ ) in the charge-starved gap zones the uncanceled field rapidly accelerates $e+/ e-$ to highly relativistic energies so that these $\gamma$-ray producing particles also follow the magnetic field lines.

As has been well established, starting with Morini (1983) and discussed explicitly by Romani \& Yadigaroglu (1995), the combination of field line sweep back, co-rotation of the radiating particles and travel time across the light cylinder tends to pile up the emission into 'caustics' in pulsar phase. As noted by Bai \& Spitkovsky (2009a) this reflects the singularity of the Jacobian matrix mapping from the emission surface to the antenna pattern on the sky. The singularity naturally produces sharp pulses with emission from a range of magnetospheric altitudes, a property shared by all competitive magnetospheric emission models. The sharpness of the peaks of the pulsed emission (Abdo et al 2009b) implies that the 'width' of the radiating zone is modest, at least in a time averaged sense. However, the resolved rise to peaks of the observed $\gamma$-ray pulses suggest either a wide range of emission height, a fundamental radiation pattern with wings, or a dispersion in the direction of the emission zone, again in a time-averaged sense.

Once one chooses a prescription for the magnetic field structure, one can follow radiation from the active emission zone to a $(\phi, \zeta)$ observer plane, where $\phi$ is the pulsar spin phase, with phase zero when the surface magnetic dipole axis passes closest to the observer line of sight. The observer line of sight is at viewing angle $\zeta$ to the spin axis. This two dimensional intensity map is the 'antenna pattern' for the pulsar radiation (Watters et al 2009). Once one specifies the inclination $\alpha$ of the magnetic dipole axis to the rotation axis one has a complete geometrical description. Of course, the choice of the radiating zone and the (energy-dependent) intensity weight through that emission zone depend on the radiation physics (Romani 1996). In practice, we use simplified assumptions that capture the basic behavior, and look for departures from the resulting predictions as guides to improved modeling of the physics. For example, it is often assumed that the gaps self-limit to a fixed potential drop (Arons 2006) which means that the $\gamma$-ray luminosity will be proportional to the open zone current (Harding 1981). One natural way to do this is to imagine radiation reaction-limited particles passing through a charge-starved gap spanning a fraction $w$ of the open zone of the pulsar magnetosphere. In this paper we take the emission surface to be relatively thin, lying a fraction $w$ of the way from the last closed field line surface to the magnetic axis. For $w$ not too close to one, this approximates the constant voltage limit if one takes the heuristic $\gamma$-ray luminosity

$$
L_{\gamma} \approx\left(10^{33} \mathrm{erg} / \mathrm{s} \dot{E}_{S D}\right)^{1 / 2} \text {. }
$$

This implies an efficiency $\eta=L_{\gamma} / \dot{E}_{S D}$ equal to the fractional gap width $w$. Realistic models must, of course, have a finite thickness for the emission zone. We find that this produces relatively small perturbations to the light curve shape if $\delta w \lesssim w$

The phase-averaged $\gamma$-ray flux for the Earth line-of-sight $F_{\text {obs }}$ is related to the total $\gamma$-ray luminosity by

$$
L_{\gamma}=4 \pi f_{\Omega} F_{o b s} D^{2}
$$

where $D$ is the distance to the pulsar (Watters et al 2009) and $f_{\Omega}$ is the 'flux correction factor'; an isotropic emitter corresponds to $f_{\Omega}=1$. Notice that we need this quantity to infer the actual pulsar $\gamma$-ray efficiency

$$
\eta=4 \pi f_{\Omega} F_{o b s} D^{2} / \dot{E}_{S D},
$$

for comparison with model predictions. A prescription for $f_{\Omega}$ is given in Equation (4) of Watters et al (2009); the solid angle corrections are applied correctly in the values in that paper (albeit for the 'Atlas'-style beam computation).

\subsection{Magnetic Field Structures}

To start, one must choose a field line structure. In the earliest light curve modeling (Morini 1983; Chiang \& Romani 1994) static dipole magnetic field lines were assumed. Emission was assumed beamed along these field lines, which were taken to occupy the rotating frame, and the resulting aberrations and time delay formed the observed pulse. While this "static" (Stat) model had the right topology (closed zone plus flaring open zone), and already predicted (for outer magnetosphere emission) the generic double $\gamma$-ray pulse lagged from the low altitude radio pulsations, it clearly is not a physically consistent picture.

The next step, introduced by Romani and Yadigaroglu (1995) and used widely by other modelers, is to use the retarded dipole instead of the static case. This is commonly referred to as the Deutsch (1955) field approximation. However, at large radius from the star, this is effectively just a rotating point dipole which is most simply expressed by Kaburaki (1980) and can be re-written as

$$
\begin{gathered}
\vec{B}=-\left[\vec{m}\left(t_{r}\right)+r \dot{\vec{m}}\left(t_{r}\right)+r^{2} \ddot{\vec{m}}\left(t_{r}\right)\right] / r^{3} \\
+\hat{r}\left(\hat{r} \cdot\left[3 \vec{m}\left(t_{r}\right)+3 r \dot{\vec{m}}\left(t_{r}\right)+r^{2} \ddot{\vec{m}}\left(t_{r}\right)\right] / r^{3}\right) \\
\vec{m}\left(t_{r}\right)=m\left(\sin \alpha \cos \omega t_{r} \hat{x}+\sin \alpha \sin \omega t_{r} \hat{y}+\cos \alpha \hat{z}\right)
\end{gathered}
$$

with $m$ the dipole magnetic moment evaluated at the retarded time $t_{r}=t-r / c$. This is the field structure used in (Watters et al 2009) and so we refer to it as the "atlas" (Atl) field. It has the virtue of having the intuitively expected 'sweepback' as one approaches the light cylinder (Figure 1).

This field is stationary in the non-inertial frame rotating with respect to the lab frame, where particles are assumed to follow the field lines. In Watters et al (2009) and earlier computations by a number of groups, the particle velocity in the lab frame was incorrectly computed as a co-rotation induced 
boost to a relativistic particle following the rotating field lines. Bai \& Spitkovsky (2009a) correctly point out that the particle velocities in the two frames are instead connected by a simple coordinate transformation. These authors assumed that the earlier computations (incorrectly) used the field (4) in the rotating frame. The boost error of Watters et al (2009) is conceptually different, but mathematically equivalent to this assumption. In practice, the coordinate transformation allows one to compute the velocity $\beta_{\|}^{\prime}$ along the magnetic field line for the particle forced to co-rotate with the star at a highly relativistic lab frame velocity $\left|\beta_{0}\right| \longrightarrow 1$

$$
\vec{\beta}_{0}=\beta_{\|}^{\prime} \hat{B}+\vec{\Omega} \times \vec{r} / c
$$

which gives

$\beta_{\|}^{\prime}=-\hat{B} \cdot(\vec{\Omega} \times \vec{r} / c)+\left\{[\hat{B} \cdot(\vec{\Omega} \times \vec{r} / c)]^{2}-(\vec{\Omega} \times \vec{r} / c)^{2}+1\right\}^{1 / 2}$

so that substituting into Equation (5) gives the direction of the particle as viewed in the lab frame. Note that $\beta_{\|}^{\prime}$ can become very small for particles traveling nearly along $\vec{\Omega} \times \vec{r}$ for $r_{\perp} \sim$ $r_{L C}$, i.e. nearly tangent to the light cylinder in the 'forward' direction. In this case small effects of particle inertia or field line perturbations will 'break open' such field lines and the particles should leave the light cylinder and contribute little to the pulse emission. Although we do not follow these physical effects, such field lines do not affect our light curves, since we taper the emissivity (see $\S 2.4$ below).

In any case, to have the plasma static in the rotating frame requires the presence of the co-rotation charge density that produces the lab frame field

$$
\vec{E}=(\vec{\Omega} \times \vec{r}) \times \vec{B} / c .
$$

This is assumed present in the 'Atlas' and similar computations, and is similarly assumed here. We refer to the revised computation as the 'pseudo force-free' (PFF) case, since it contains co-rotation enforcing charges, but no currents. These PFF computations, for both TPC and OG-type models, give light curves slightly distorted from in the 'Atlas'-type computations and appear to match well to the sample antenna patterns and light curves of Bai \& Spitkovsky (2009a). The principal effect, as noted by these authors, is that for small $w<0.02$ the low altitude emission that dominates the second pulse of the TPC model has decreased phase folding, making the Jacobian non-singular. There is a pulse peak but without a true caustic there is no sharp pulse. This also weakens somewhat the first TPC peak for such high $\dot{E}$ pulsars. For pulsars with larger $w$ and for the higher altitude emission of the OG model there are departures from the 'Atlas'-style computation, but the effects are more subtle. In the Appendix we present arrays of example light curves for the TPC and OG models. These may be compared with, and considered as updates to, the similar light curve panels in Watters et al (2009). We also update the figures describing the pulse width and flux correction factors and their variation with viewing geometry, for comparison with that paper.

\subsection{Current-induced B Perturbations}

The gap-accelerated radiating charges alone produce some currents in the open zone, and we expect the gap-closing pair front to produce densities comparable to the co-rotation value for at least some of the open field lines. This current will perturb the magnetic field of Equations (4).

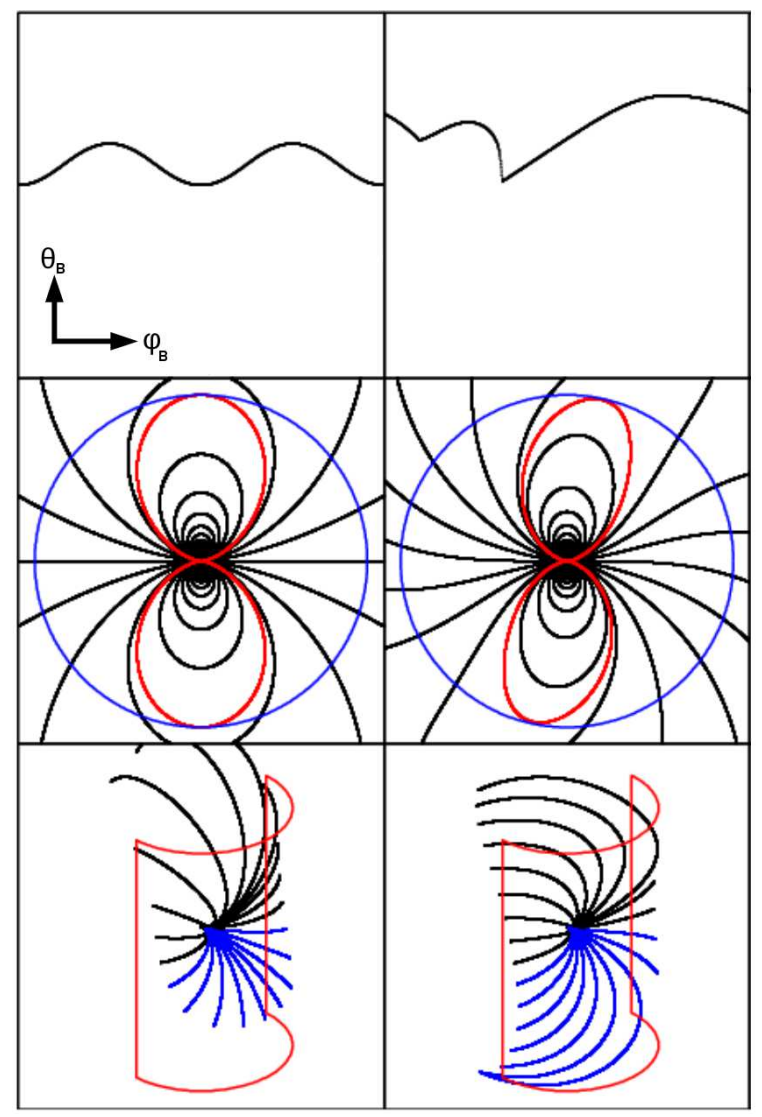

FIG. 1.- Illustrations of the basic dipole field geometry. Left: Static, Right: retarded point dipole (as used in the 'Atl' and 'PFF' computations). Top the position of the open zone boundary (polar cap boundary) in magnetic coordinates $\theta_{B}\left(\phi_{B}\right)$ for magnetic inclination $\alpha=70^{\circ}$. Middle - magnetic field lines in the equatorial plane for $\alpha=90^{\circ}$. Bottom - field lines for $\alpha=80^{\circ}$ above one polar cap with lighter (blue) showing the lines which cross $r_{N C}$, the 'Outer Gap' lines.

In the spirit of attempting analytic amendments to the vacuum model, we applied the approximate perturbation field computed by Muslimov and Harding (2009) for a pair-starved current flow in the open zone. This is expressed in magnetic coordinates $\left(r_{B}, \theta_{B}, \phi_{B}\right)$, where the perturbation amplitude $\epsilon^{\prime}$ determines $B^{\prime}=\epsilon^{\prime}\left[2 m \Omega /\left(r^{2} c\right)\right]$ with $m$ the magnitude of the star's dipole moment

$$
\begin{gathered}
B_{r_{B}}^{\prime}=B^{\prime} s\left(1-s^{2}\right)^{-1} \\
B_{\theta_{B}}^{\prime}=B^{\prime}\left[\left(1-s^{2}\right)^{-1} \frac{\partial s}{\partial \theta_{B}}+\left\{\sin \theta_{B}\left(1-s^{2}\right)^{3 / 2}\right\}^{-1} \frac{\partial s}{\partial \phi_{B}}\right] \\
B_{\phi_{B}}^{\prime}=B^{\prime}\left[-\left(1-s^{2}\right)^{-3 / 2} \frac{\partial s}{\partial \theta_{B}}+\left\{\sin \theta_{B}\left(1-s^{2}\right)\right\}^{-1} \frac{\partial s}{\partial \phi_{B}}\right]
\end{gathered}
$$

and $s=\cos \theta_{\text {rot }}=\cos \alpha \cos \theta_{B}+\sin \alpha \sin \theta_{B} \cos \phi_{B}$. This perturbation field is defined with respect to the static vacuum dipole. We take $\epsilon^{\prime}$ positive for outward-flowing electrons (i.e. negative current) above the magnetic pole passing closest to the Earth line-of-sight. This pole is generally inferred to produce the low-altitude radio pulsar emission. If this pole has the opposite current (i.e. opposite magnetic polarity) we have $\epsilon^{\prime}<0$. Of course an electrodynamically self-consistent model will have a particular sign of $\epsilon^{\prime}$ for, e.g. the positive magnetic pole, but our PFF model includes no current and so 
is insensitive to the sign of $B$; only this perturbation current breaks the degeneracy.

To approximate the retarded solution, we matched this perturbation to our magnetosphere, mapping $B\left(r_{B}, 0, \phi_{B}\right)$ to the magnetic axis of the swept-back dipole. The perturbation field becomes singular along the rotation axis where $s \longrightarrow \pm 1$. By exponentially tapering this singularity $(\propto$ $1-e^{(|s|-1) / \sigma_{s}}$, with $\left.\sigma_{s}=0.25\right)$ we were able to integrate the field lines to determine the last closed field line surface (see below). Unfortunately, for lines near $\phi_{B}=0, \pi$ the residual effects of this singularity still dominate, making the cap boundary at these azimuths unstable (Figure 2).

Accordingly we proceeded with a simpler toy perturbation field computed by integrating a constant line current passing through the star along the magnetic axis (the current extends to cylindrical radius $r_{\perp}=r \sin \theta=1.2 R_{L C}=1.2 \mathrm{c} / \Omega$ to minimize end effects). This perturbation field

$$
\vec{B}^{\prime}(\vec{r}) \propto \int_{B\left(r_{b}, 0, \phi_{B}\right)} \mathrm{d} \vec{I} \times \vec{r}
$$

is treated as static in the rotating frame and is summed with the field of (4). The effect of this simple perturbation field is dominated by the twisting of field lines along the magnetic axis, as can be seen by the shift of the 'notch' foot-points of the last closed field line cap boundaries in Figure 2. This rotation is also present in the Muslimov and Harding (2009) field, but is dominated by the singularity effects except for nearly aligned rotators $\alpha \approx 0$. For ease of comparison, we scale the perturbation amplitude for fields (8) and (9) to the underlying dipole field (4) so that

$$
\epsilon=\left\langle B^{\prime}\left(0.5 r_{L C}, \theta, \phi\right)\right\rangle /\left\langle\left|B\left(0.5 r_{L C}, \theta, \phi\right)\right|\right\rangle
$$

where we average over a sphere at $r=0.5 r_{L C}$. Again the sign of $\epsilon$ is determined by the current; we expect opposite values for the two poles.

None of these fields is fully realistic. In particular the current-induced $B$ field perturbations used above are not complete consistent field/current systems. Nevertheless, given that the vacuum field geometry has already provided encouraging successes in approximating the observed light curves, it is useful to explore how current systems can perturb the modeled beam patterns and pulse shapes. As an example, Figure 3 shows the 'antenna patterns' in the hemisphere containing emission from above the null charge surface (the OG case below). The unperturbed beam pattern (middle) suffers opposite distortions in the two hemispheres.

\subsection{Gap Locations}

For each field structure, we assume the appropriate corotation charge density so that the field pattern is stationary in the rotating frame. We define the open zone by identifying field lines tangent to the light cylinder (actually at slightly smaller radius for numerical expediency). These field lines are traced back to the surface where they define the open/closed-zone boundary. In Figure 1 we show the foot-point locations, equatorial plane field lines (for $\alpha=90^{\circ}$ ) and the last closed field line structure for an inclined $\alpha=80^{\circ}$ rotator. Our radiating surface is always in the open zone toward the magnetic axis from this boundary. This plot shows the static ('Stat') and point retarded dipole ('Atl' \& 'PFF') field structures. The active field lines for the outer gap are lighter (plotted in blue).

With the emitting field lines defined, we must choose the extent of the radiating surface. For the TPC model,

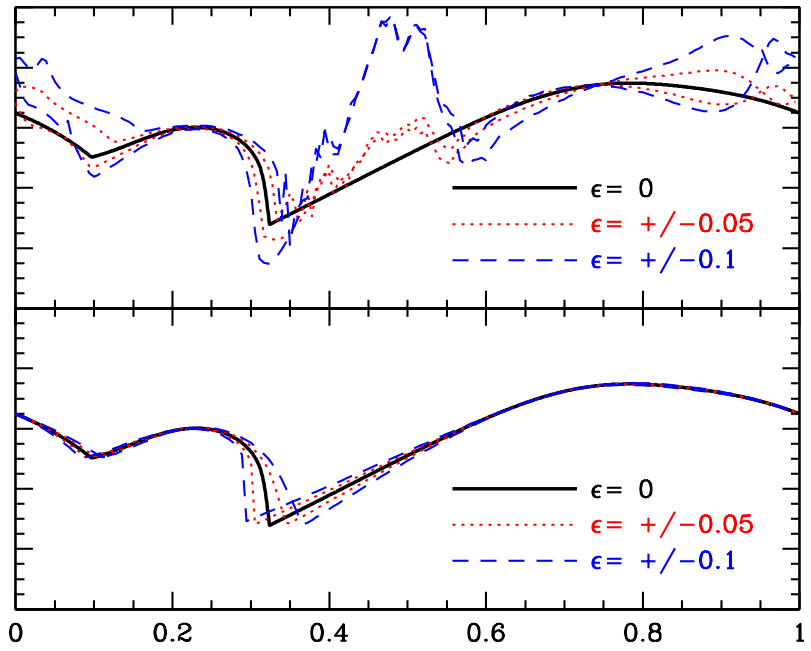

FIG. 2.- Open zone boundary (polar cap) foot-points: magnetic colattitude $\theta_{B}$ on the star surface as a function of magnetic azimuth $\phi_{B}$ for the $\alpha=70^{\circ}$ PFF field with current-induced perturbations. Top: the Muslimov and Harding (2009) pair-starved field, mapped to the retarded dipole. Bottom: retarded dipole with perturbations from magnetic axis line currents. Notice that the currents 'twist' the field lines for both models, shifting the 'notch' at $\phi \approx 0.3$. Although the singularity in the Muslimov and Harding (2009) field (upper panel) has been smoothed, we still see dramatic instability for lines directed near the rotation axis $\left(\phi_{B} \approx\right.$ $0.4-0.6$ and $\phi_{B} \approx 0.8-0.1$ ).

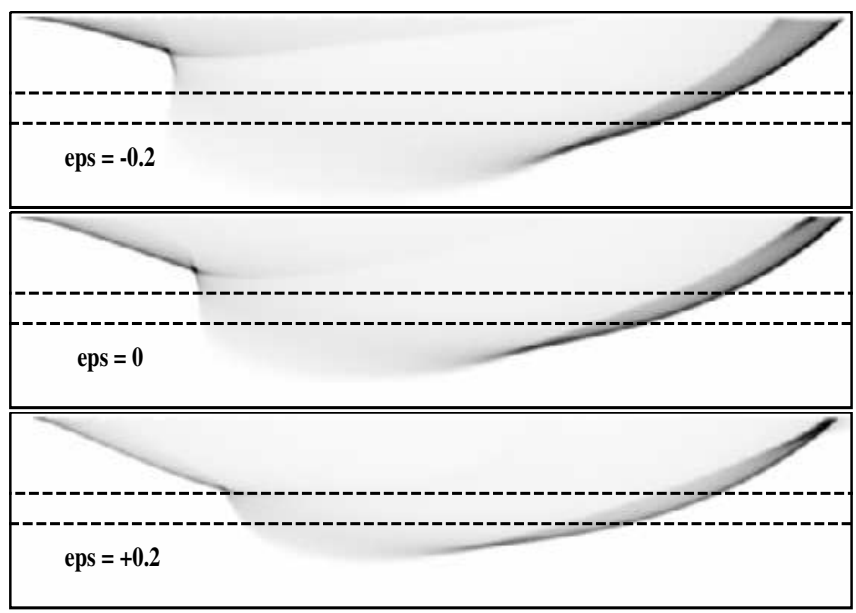

FIG. 3.- Antenna patterns for current-induced perturbations. Pulsar light curves are obtained from horizontal cuts across these images, after normalization by $[\sin \zeta]^{-1}$. Here we show emission from above the null charge surface (OG case) for $\alpha=60^{\circ}, w=0.1$. The middle panel shows the unperturbed PFF structure. The upper $(\epsilon<0)$ and lower $(\epsilon>0)$ panels show the effect of a perturbing line current along the magnetic axis. Note that the front caustic is better closed for $\epsilon>0$, but for negative $\epsilon$ the 'notch' structure is more pronounced. The dashed lines show the range where the viewing angle $\zeta$ passes close to the low altitude emission from the opposite magnetic pole, i.e. when one expects a radio pulse. Measurement of light curve perturbations can thus determine the sign of $B$ for a given magnetic pole.

we follow the original definition (Dyks and Rudak 2003; Dyks, Harding, and Rudak 2004), taking the emission surface to be the full set of last-closed field lines, but stopping emission once the radial distance is $r>r_{L C}$ or once the distance from the rotation axis exceeds $r_{\perp}>0.75 r_{L C}$. This cut-off is required to avoid the higher altitude pulse components (as used in the OG picture), otherwise the modeled light curves generally have three or four pulse components. As in Watters et al (2009) we make this model more physical by placing the emission surface in the open zone, separated from 
the last closed surface by the thickness $w$ appropriate to the pulsar $\dot{E}$ (Equation 1).

In the outer gap (OG) model radiation is produced on field lines crossing the null-charge surface $\left(\vec{\Omega} \cdot \vec{B}\left(R_{N C}\right)=0\right)$, with emission starting near this crossing point and extending toward the light cylinder. Again, we assume a gap thickness $w$. Field lines that do not cross the null charge surface before $r_{L C}$ do not radiate.

The outer gap emission should be dominated by a section of the flared cone of field lines above a given pole. For these simple vacuum-based models, some field lines just inside the last closed surface extend for a very long distance before exiting the light cylinder or crossing the null charge surface. For example, some field lines arc over the star and cross $\vec{\Omega} \cdot \vec{B}=0$ for the first time far from the star with a path length (in units of $\left.R_{\mathrm{LC}}\right) s \gg 1$. These field lines define a disjoint, 'high altitude' second gap surface, that does not in most cases appear to be active (although the HF pulse components of the Crab might represent such emission; Moffet \& Hankins 1996). Physically, we expect that such lines arcing near $R_{L C}$ will be opened in a real magnetosphere with currents and particle inertia. Also, gaps on field lines starting at large $r_{N C}$ will likely be inactive if the soft emission needed to close the gap arises from other field lines at much smaller $r_{N C}$. Accordingly, as in Romani and Yadigaroglu (1995), we apply a path-length $s$ cutoff in the gap surface emissivity. Here the functional form used (for $s>1+2 s_{\mathrm{NC}, \min }$ ) is

$$
F(s) \propto e^{-\left[\left(s-2 s_{\mathrm{NC}, \min }-1\right) / \sigma_{s}\right]^{2}}, \quad \sigma_{s}=0.1
$$

with $s_{\mathrm{NC}, \min }$ the lowest null charge crossing for any active field line and all distances in units of $R_{L C}$. A detailed surface emissivity weighting awaits a physically realistic spectral emission model.

While this work was being prepared for publication, a new discussion of light curve formation in fully force-free models has been presented by Bai \& Spitkovsky (2009b). These authors note the limitations with pure vacuum modeling mentioned above. They also argue that in fully force-free magnetospheres if the radiation is associated with the return current sheet, neither TPC nor OG pictures produce realistic light curves, and propose a new 'Annular Gap' (AG) geometry that has emission extending beyond the light cylinder. The footpoints of the AG field lines are the same as those used here for the TPC geometry (for a given $w$ ) but in Bai \& Spitkovsky $(2009 \mathrm{~b})$ the co-rotating pulsed emission is taken to extend well beyond the the light cylinder. Thus, this model covers our OG zone plus additional emission at both high and low altitudes. As we see below, the vacuum dipole field (possibly with current-induced B perturbations) does a rather good job of matching the observed pulsations - it will be interesting to see if the force-free numerical fields using the AG or OG geometries can match the data comparably well.

\subsection{Computational Grid}

We identify the last closed field line surface (those field lines tangential to the speed of light cylinder) and trace these field lines to the polar cap surface at $r_{*}$. We follow the field line structure to $<10^{-4} r_{L C}$, allowing good mapping even for pulsar periods of $\geq 1 \mathrm{~s}$. A single magnetosphere model can serve a range of pulsar periods, since the OG and TPC model features are computed as distances in fractions of $r_{L C}$. The pulsar period only affects $R_{*} / R_{L C}$ and hence the inner boundary of the emission zone.
Models are computed for all magnetic inclinations $\alpha$. Photons are emitted along the particle path in the lab frame (moving tangent to the local field line in the co-rotating frame) and then phased, including light travel time across the magnetosphere, to add to the skymap antenna pattern, as would be seen by distant observers. We assume uniform emissivity along all active field lines (except for the taper at large pathlength $s$ as noted above). In practice we trace the trajectories for $\sim 10^{4}$ photons per field line, which produces adequately smooth light curves for the present computation. Models are computed for a range of gap widths from $w=0.01$ to $w=0.3$, to accommodate a range of pulsar $\dot{E}$.

\subsection{Lightcurve Fitting}

Our next task is to take this family of light curves and compare with the observed pulse profiles. We take our $\gamma$-ray data from the full band $E>0.1 \mathrm{GeV}$ pulse profiles published in Abdo et al (2010). These use the first six months of Fermi data and give good statistics for the relatively bright pulsars considered here. In this Fermi pulsar catalog, a background level is estimated from the surrounding sky. Interestingly, some pulsars have significant steady (DC) emission. Matching this emission is an important test of the model - accordingly we fit to the total emission, pulsed and un-pulsed, after subtracting the background, as estimated in Abdo et al (2010). In particular, we do not fit the un-pulsed flux as a separate degree of freedom - the excess above the LAT pulsar catalog background is determined by the magnetosphere model.

We take the measured pulsar $\dot{E}$ and compute $w$ according to Equation (1), then use the closest value from the model grid. For this grid we take all $\alpha$ (in one degree intervals) and fit the model light curve for each $\alpha$ and $\zeta$. To compare with the data we bin a given model curve into the phase bins ( 25 or 50/cycle) defining the light curve for the pulsar in Abdo et al (2010). The total model counts are normalized to the background-subtracted data counts and we then search for the best-fit model to the combined (pulsar plus background emission) light curve.

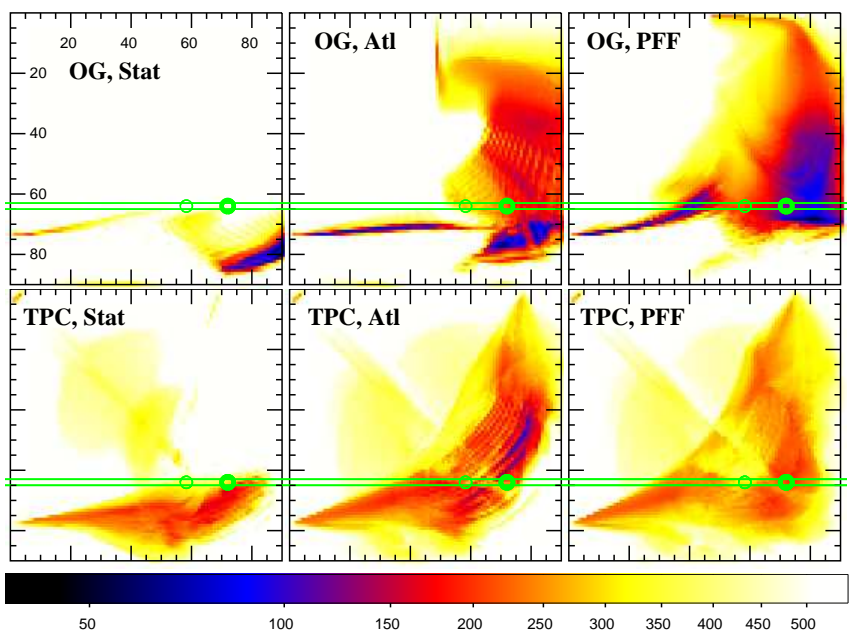

FIG. 4.- Goodness of fit $\chi(\alpha, \zeta)$ surface for Vela with several models. Low $\chi$ (dark) represent the best fits. The three columns give results (Left to Right) for Stat, Atl and PFF field structures. Top are OG models, bottom are TPC. The green lines show $\tilde{\zeta}$ allowed by $C X O$ PWN fitting. The ellipses give the combined constraints including radio polarization data.

A variety of fitting statistics work well. Simple $\chi^{2}$ fitting generally finds the best solutions, but is controlled by the more numerous off-pulse and 'bridge' emission phase 


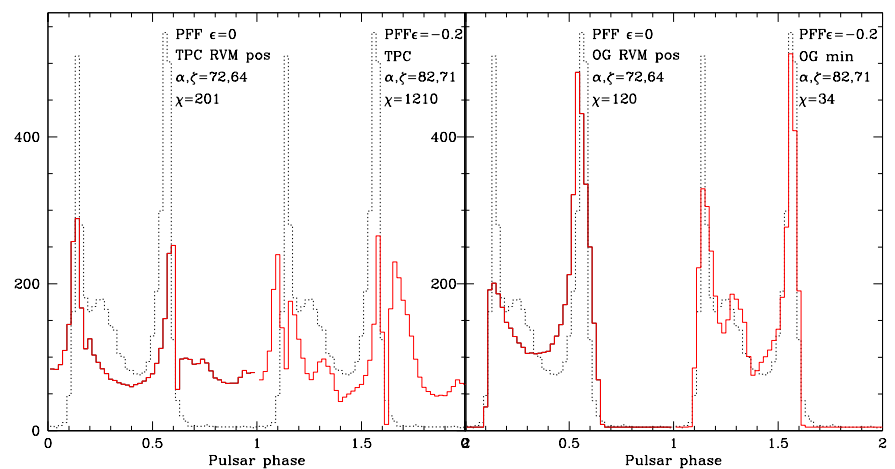

FIG. 5.- Pulse profiles for the TPC (left) and OG (right) models for Vela using the PFF field structure. The dotted pulse profiles show two periods of the LAT $(E>0.1 \mathrm{GeV})$ Vela data from ?. The first model pulse in each panel is for the PWN/RVM-fit $\alpha, \zeta$, with no current perturbation while the second is for the parameters of the global OG fit minimum. The TPC fit quality varies only weakly with angle and does not improve substantially away from the RVM values.

bins and so occasionally provides poor discrimination against models with unacceptably weak peaks. Choosing the model with the smallest maximum model-data difference for any bin $\operatorname{Max}\left[\operatorname{Abs}\left(\operatorname{Mod}_{i}-D_{i}\right)\right]$ does an excellent job of matching the peaks, but less well on the bridge flux. We find that an exponentially tapered weighting of the largest model-data differences is robust and produces sensible results. Thus we minimize

$$
\chi_{n}=\frac{\left(\operatorname{Mod}_{\mathrm{i}}-\mathrm{D}_{\mathrm{i}}\right)^{2}}{D_{i}} \mathrm{e}^{-i\left(\left|\operatorname{Mod}_{\mathrm{i}}-\mathrm{D}_{\mathrm{i}}\right|\right) / n}
$$

with $i\left(\left|\operatorname{Mod}_{\mathrm{i}}-\mathrm{D}_{\mathrm{i}}\right|\right)$ the index of the phase bin differences, sorted large to small. Small $n \ll 1$ just uses the biggest difference bin (and thus focuses on the peaks), large $n$ goes over to classical $\chi^{2}$ weighting. The method is only weakly sensitive to $n$ and an e-folding weighting $n \approx 3$ worked well.

Our reference phase $\phi=0$ occurs when the dipole axis, spin axis and Earth line of sight all in the same plane. It is generally assumed that radio emission arises at relatively low altitudes (but see Karastergiou and Johnston 2007), and that the radio pulse occupies a fraction of the open zone field lines at low altitudes. In Abdo et al (2010) phase zero is taken to be the amplitude peak of the main radio pulse. However, the radio pulse profile often exhibits 'patchy' illumination of the emission zone (Lyne and Manchester 1988). Thus the radio peak may not mark the phase of the closest approach of the magnetic axis. Additional geometrical information comes from the linear polarization sweep of the radio pulse, with the maximum sweep rate associated with the line-of-sight passage past the magnetic axis. This maximum often occurs well away from the intensity peak. Finally, if the radio emission arises well above the star surface, relativistic effects can perturb the phases of the closest approach (ideally pulse intensity maximum) and sweep rate. Blaskiewicz, Cordes \& Wasserman (1991) argue that the approximate phase shifts are

$$
\phi_{\text {Int }} \approx-r / r_{L C} \quad \phi_{\mathrm{Pol}} \approx+3 r / r_{L C} .
$$

Thus polarization information can give additional constraints on the absolute phase, but altitude (plus mode changing and other sweep perturbations) can lead to substantial additional uncertainty. Accordingly, we have the option to fit light curves with the model phase free. Generally, we allow $\phi$ to shift by as much as \pm 0.1 of a rotation, and retain the best fit model. When no radio pulse is known we allow full $\delta \phi= \pm 0.5$ freedom in the pulse phase. The phase for the best-fit model is recorded in the $(\alpha, \zeta)$ map, as well as the model light curve and properties of the pulsed emission, for use in further analysis. Recalling that the radio phase $\phi=0$ in Abdo et al (2010) is set at the pulse intensity peak, we expect that our fit, which estimates $\phi_{0}$ for the true magnetic axis, will tend to give small $\delta \phi>0$ for emission from finite pulse heights. However, we expect that $\delta \phi$ will be less than $\phi_{\mathrm{Pol}}$. In practice, patchy pulses and polarization sweep uncertainties make $\delta \phi$ of any modest amplitude plausible.

In considering the values of the fit statistic $\chi_{n}$, we must remind the reader that these are not complete models. Detailed radiation physics will certainly change the light curve shape from the uniform weighting assumed here. Also, the lack of self-consistent magnetospheric currents must, at some level, cause light curve shape errors. This, coupled with the excellent signal-to-noise of the Fermi light curves guarantees that these will not be statistically "good" fits. Monte Carlo experiments with the $\chi_{n}$ fit statistic show that for Poisson realizations of a 50 bin light curve, the typical value is $\left\langle\chi_{3}\right\rangle=7.5$ while $99 \%$ and $99.9 \%$ values are 15.5 and 19 . Thus fit differences of $\Delta \chi_{3} \approx 8$ are statistically significant at the normal $2.5 \sigma$ level. This gives a guide to interpreting the relative goodness of fits to the various models.

\section{FIT EXAMPLES}

We start with the archetype $\gamma$-ray pulsar, Vela. For this pulsar we show the fit statistic $\chi$ surfaces for the three approximate dipole field structures (Stat, Atl, PFF) discussed above, with darker colors indicating better model fits. The upper row is for OG models, the lower row for TPC. For OG all field structures show a similar topology, with best-fit solutions at $\zeta \approx 70-80^{\circ}$ and $\alpha \approx 60-90^{\circ}$, and no solutions at small $\alpha, \zeta$. The swept-back models (Atl and PFF) have a tail of solutions to smaller $\alpha$. The TPC scenario can produce models for nearly all $\alpha, \zeta$. However, except for the 'Atlas' geometry which, as also noted by Bai \& Spitkovsky (2009a), has unrealistically sharp caustic pulses for small gap width $w$, the models produce much poorer fits than the OG scenario, as they have weak pulses and far too much off-pulse (DC) emission.

\subsection{External Angle Constraints}

The model fits in $(\alpha, \zeta)$ space are especially useful when we have external constraints on these angles. Some care is needed, however, in applying angle constraints from other wavebands. For Vela and a number of other young pulsars with bright X-ray pulsar wind nebulae (PWNe) we have been able to place relatively model-independent constraints on viewing angle by fitting for the inclination $\tilde{\zeta}$ of the relativistic Doppler-boosted PWN torus ( $\mathrm{Ng}$ and Romani 2008). This method does not, however, distinguish the sign of the spin axis. Therefore the X-ray torus fits allow two possible viewing angles between the positive spin axis and the Earth line-of-sight $\zeta=\tilde{\zeta}$ and $\zeta^{\prime}=180^{\circ}-\tilde{\zeta}$. In fact, in $\mathrm{Ng}$ and Romani (2008) the torus position angle was restricted to be $0^{\circ} \leq \Psi<180^{\circ}$, so the angles quoted in this paper may represent either $\zeta$ or $\zeta^{\prime}$.

In the context of the rotating vector model (Radhakrishnan and Cooke 1969) radio polarization data can also constrain the viewing angles. In most cases, the small range of phase illuminated by the radio pulse allows only an estimate of the magnetic impact parameter

$$
\beta=\zeta-\alpha \approx \sin ^{-1}\left[\sin \alpha /(\mathrm{d} \Psi / \mathrm{d} \phi)_{\max }\right]
$$




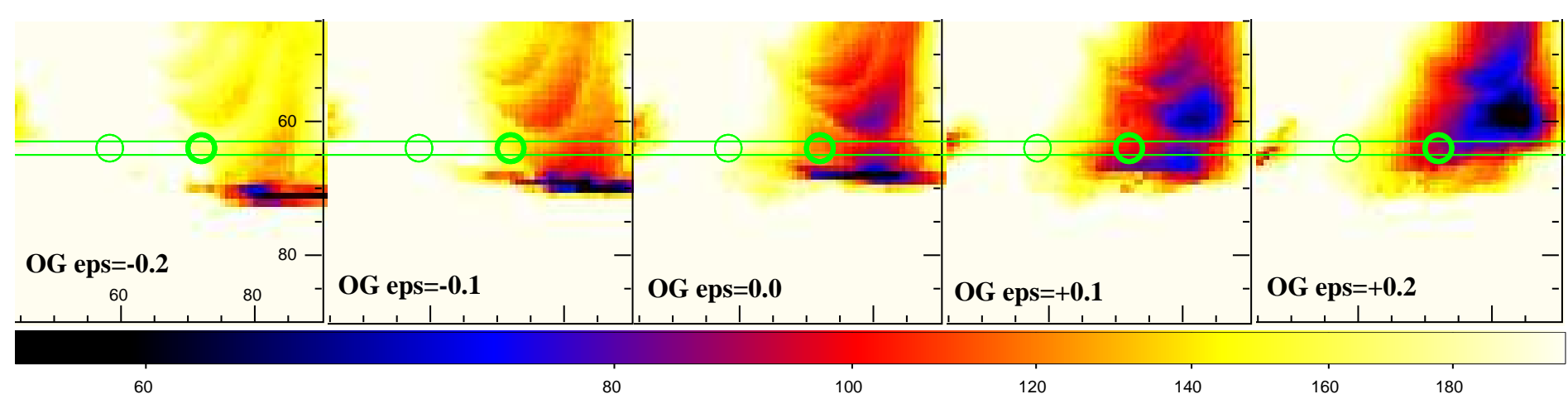

FIG. 6.- Comparison of the lower right quadrant of the OG fit planes for models with magnetic axis current-induced $B$-field perturbations. Left to right: $\epsilon=-0.2,-0.1,0.0,+0.1,+0.2$. The plots show the range $45^{\circ} \leq \alpha \leq 90^{\circ}, 45^{\circ} \leq \zeta \leq 90^{\circ}$.

where the maximum rate of the polarization position angle sweep $\Psi(\phi)$ occurs at $\phi_{\text {pol }}=0$, near the closest approach to the magnetic axis. Here the sign of the sweep is meaningful, determining whether the line of sight is closer to or farther from the positive rotation axis than the observed magnetic pole (at inclination $\alpha$ ). Thus, when we have an independent PWN estimate of $\tilde{\zeta}$, we have two possible $\alpha$ values - e.g. for a negative sweep we get $\beta<0$ and $\alpha=\zeta-\beta$ or $\alpha=\zeta^{\prime}-\beta$. In this paper, we tabulate the possible values of $\zeta$ and $\zeta^{\prime}$, but save space by plotting only in the positive rotation hemisphere. Thus there are two values possible for $\alpha$ from Equation (10), but when $\alpha$ or $\zeta>90^{\circ}$, we reflect back into the positive hemisphere for plotting purposes.

Occasionally, when the radio pulse is very broad or when the pulse profile presents an interpulse, the radio polarization can make meaningful estimates of both $\alpha$ and $\zeta$, from fits to the full polarization sweep

$$
\tan \left(\Psi+\Psi_{0}\right)=\frac{\sin \alpha \sin \left(\phi-\phi_{0}\right)}{\sin \zeta \cos \alpha-\cos \zeta \sin \alpha \cos \left(\phi-\phi_{0}\right)}
$$

where the polarization has the absolute position angle $\Psi_{0}$ at $\phi_{P o l}=\phi_{0}$. Keith et al (2009) have recently presented several examples. When the radio illumination is insufficient for a full solution, we can still break the degeneracy of the fits using the X-ray $\tilde{\zeta}$ constraints. Finally, if the magnetic inclinations orientations are, at least initially, isotropic we expect the inclination choice giving $\alpha$ closest to $90^{\circ}$ to be preferred on statistical grounds.

In Figure 4 the two possible RVM $\alpha$ solutions for Vela (Johnston, et al 2006) consistent with the PWN data are shown by the green circles. Note that the OG and TPC models have minima close to the solution at larger $\alpha$ (bold circle). In general, with constrained $\alpha, \zeta$ values one can make a discrimination between the models. Note that for the OG model the position of the minimum is closest to the preferred angle for the relatively physical 'PFF' case. The values of the fit statistic are substantially worse for TPC at all locations near these preferred angles (Table 1). Figure 5 shows the PFF light curves at the RVM angles, and at the global fit minimum, for the TPC and OG scenarios.

\subsection{Testing Field Perturbations}

The best-fit models in Figure 4 are several $\sigma$ off of the PWN/RVM angles. This suggests either that there are systematic errors in these angle estimates or that the true magnetosphere structure is slightly different than the PFF estimate above. The measurement of such offsets for a number of pulsars should enable us to map the required geome- try differences and test physical models for their origin. As an example, here we illustrate the perturbing effect of magnetospheric currents. Figure 6 shows the $\chi$ plane for fits including a magnetic axis current (Equation 9). The current shifts the fit minimum and distorts the light curves. For a best fit in the RVM-determined region positive currents are required for the OG model. The best models within $\sim 1 \sigma$ of the PWN+RVM angles also prefer a small positive current. For negative $\epsilon$ the best fit region shifts further from the externally determined angles, although the match to the pulse shape is quite good, reaching a global minimum $(\chi=34)$ at $(\alpha, \zeta)=\left(82^{\circ}, 71^{\circ}\right)$ for $\epsilon=-0.2$. Since the global minimum does not shift precisely to the PWN/RVM angles, we infer that (unsurprisingly) the true field geometry is only approximated by this simple model. The TPC model fits are not improved by including finite $\epsilon$; the best minimum anywhere near the external PWN/RVM angles has $\chi \approx 200$.

To summarize, for Vela with the external constraint, PFF is clearly preferred and the best fit models are relatively close to the known $\alpha, \zeta$. The rotation axis is inferred to be oriented out of the plane of the sky, we are viewing at $\zeta \approx 65^{\circ}$ and the magnetic inclination is large at $\alpha \approx 75^{\circ}$. Positivecurrent field perturbations are preferred. The $\gamma$-ray pulse fits imply that $\phi_{0}$ lies earlier than the radio peak (and earlier than the maximum sweep rate). This would suggest that Vela has patchy radio emission dominated by the 'trailing' side of the radio zone.

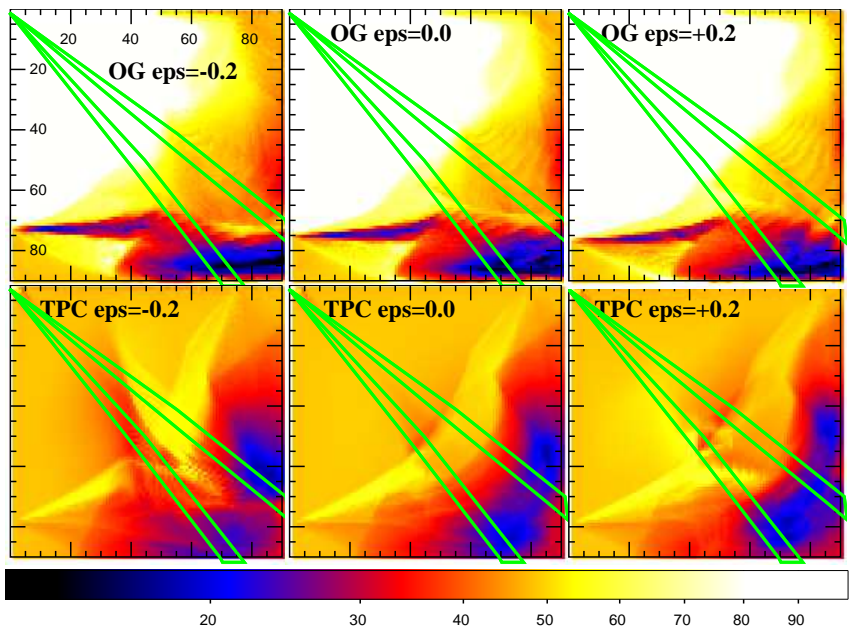

FIG. 7.- Goodness of fit surface for PFF models of PSR J1952+3252, Left to right: $\epsilon=-0.2,0.0,+0.2$, top: $\mathrm{OG}$, bottom: TPC. The regions indicated by the maximum polarization sweep rate $\mathrm{d} \Psi /\left.\mathrm{d} \phi\right|_{\max }$ are shown as two green wedges. 


\subsection{Other EGRET Pulsars}

The next most energetic pulsar in our test set is PSR $\mathrm{J} 1952+3253$. As for Vela, the three field structures give quite similar $\chi$-plane maps. In (Figure 7) we show the $\chi$ maps for the PFF model with three values of $B^{\prime}$. This pulsar has two narrow peaks separated by $\Delta=0.49$ in phase, so we find that the TPC model can produce reasonable solutions, as indicated by the relatively dark regions in the lower panels. This object is presently interacting with the shell of its supernova remnant, CTB80, and so produces a PWN bow shock rather than a torus, precluding a $\tilde{\zeta}$ measurement. However from the max sweep rate of the radio polarization from Weisberg et al. (1999), we can infer a constraint on the magnetic impact parameter (two green diagonal bands). The sweep maximum appears to lie towards the tail of the broad radio pulse, consistent with our fit $\delta \phi \approx 0.05$; again the radio pulse peak would represent patchy emission, in this case from the leading edge of the emission region at relatively low altitudes.

Both models prefer relatively large $\alpha$ and $\zeta$, and each can produce acceptable light curves within the region allowed by the radio data. If improved radio measurements or other angle constraints can be obtained, it may be possible to select between these two models for this object and also measure the altitude of the radio emission zone.

Next and only slightly less energetic is PSR B1706-44 = PSR J1709-4429. This object, in contrast to PSR $\mathrm{J} 1952+3252$, has a narrow double pulse with $\Delta=0.25$. Here OG models clearly prefer the small $\alpha$ RVM solution. While the best OG fits have $\chi \leq 30$, the TPC models in the PWN-determined $\zeta$ strip are poor with $\chi>90$. Polarization sweep data are limited for this pulsar. The published plots in Johnston, et al (2006) give $\alpha \approx 34^{\circ}$, which is best matched with negative currents $\epsilon \leq 0$. These fits however, imply a surprisingly large offset for the magnetic pole of $\delta \phi=-0.04=14^{\circ}$, which would put the magnetic axis at the leading edge of the radio pulse. Intriguingly, the best fit of all parameters is found with a OG PFF $\epsilon=0.05$ model at $\zeta=53^{\circ}, \alpha=47^{\circ}(\chi=19)$. This very good light curve (Figure 9) has the magnetic axis near the radio peak with $\delta \phi=-0.01$, but would have a polarization sweep rate $\sim 3 \times$ larger that that seen in present radio data. Again, improved radio polarization data might help shed light on the preferred inclination angle.

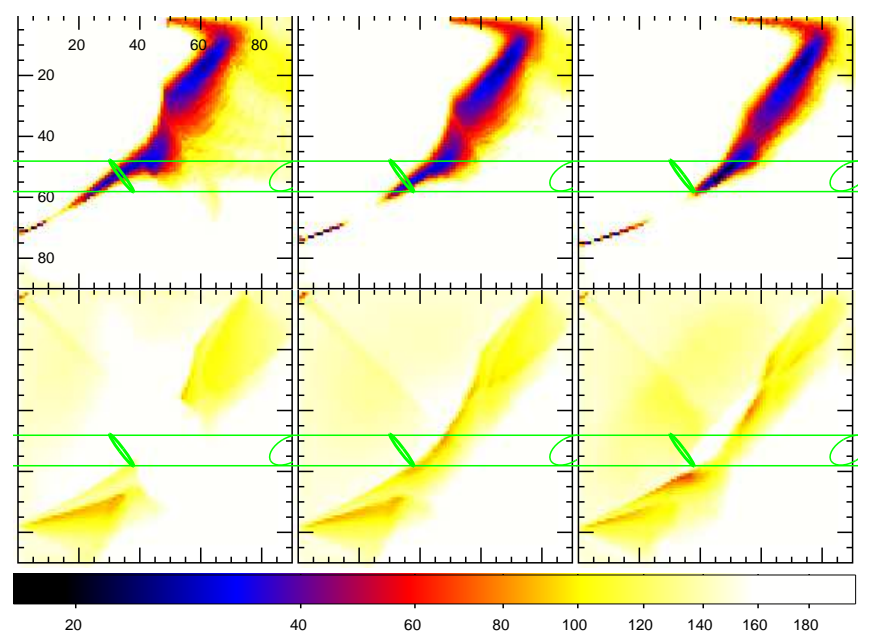

FIG. 8. - Goodness of fit surface for PSR J1709-4429, panels as for Figure 7 The $\mathrm{X}$-ray determined $\tilde{\zeta}$ is shown by the horizontal band. For this pulsar the smaller $\alpha$ RVM solution is clearly preferred.
The next pulsar, PSR B1055-52 = PSR J1057-5226, is appreciably older. It shows a pulse that is nearly a square wave in the full energy band, although the energy-dependent light curves of (Abdo et al 2010) indicate that it is likely a tight double of separation $\Delta \approx 0.2$ with strong bridge emission. The pulsar is too old to show a bright PWN, so no $\zeta$ is available from the X-rays. Luckily in the radio it has both a very broad pulse and interpulse and radio polarization fitting can strongly constrain both $\alpha$ and $\zeta$. We adopt the values from (Weltevrede and Wright 2009) and note the phase of the maximum polarization sweep is clearly at $\phi=0.08$ (later) than plotted in (Abdo et al 2010). Indeed, we find that the model fits prefer positive $\delta \phi$, although the value is slightly larger than expected. The peaks on the model light curves are also stronger than in the data for these parameters; the match can be significantly improved for models with finite $\delta w$ (not detailed here). As noted by Watters et al (2009), no viable TPC models lie anywhere near the RVM-determined angles. For OG there is a shallow fit minimum centered on the RVM angles. Superposed on this are $\chi$ stripes, caused by the relatively coarse (25 bin) LAT light curve. With improved LAT data the fits should become more discriminating.

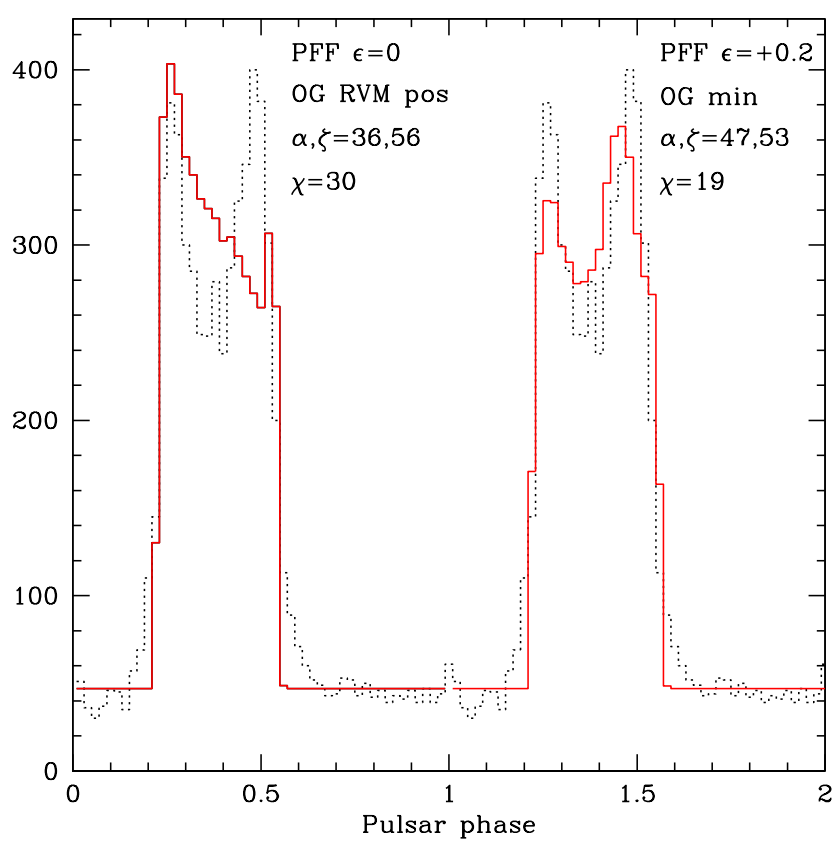

FIG. 9.- Light curves for two PFF models for PSR J1709-4429. The model at the RVM position has a rather weak second peak and requires a relatively large $\delta \phi$. A model at the PWN $\zeta$ but slightly larger $\alpha$ provides the best fit at $\delta \phi \sim 0$.

For the TPC model there is a large region with modest $\chi$ values at small $\alpha$ and $\zeta$, with the best solution listed in Table 1. However these models are not only inconsistent with the radio data, but all produce single pulses with one broad, approximately Gaussian, component and are unlikely to represent the true solution. If one allows any viewing angle, exceptionally good fits can in fact be found for OG with $\chi$ slightly below the statistical minimum value. Despite an excellent match to the observed $\gamma$-ray pulse, this is also unlikely to represent the true solution. This underlines the fact that sensible solutions must be compatible with external angle constraints and, when these constraints are applied with care, one may use the multi- 
wavelength data to rule out otherwise viable pulse models.

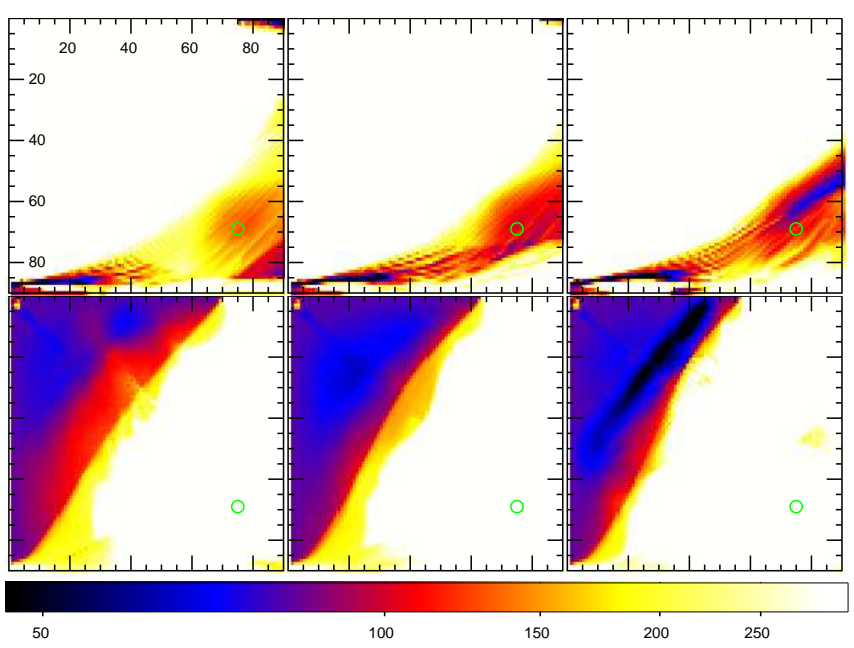

FIG. 10.- Goodness of fit surface for PSR J1057-5226, panels as for Figure 7 For this pulsar, interpulse emission allows a good RVM fit for both the inclination angle $\alpha$ and viewing angle $\zeta$ (green circle).

Finally, we discuss Geminga, the archetype of the old $\gamma$ selected pulsars, and the only one known prior to the launch of Fermi. This object does show an X-ray PWN, but like many old objects it is dominated by bow shock structure (Pavlov, Sanwal \& Zavlin 2006) and so it will be very difficult to extract torus/jet constraints on $\zeta$. However we do know that despite very sensitive radio searches, no convincing detection of pulsed radio emission has been found, implying that $|\beta|=|\zeta-\alpha|$ is large. It has also been suggested that the thermal surface pulsations are most consistent with a nearly aligned rotator (small $\alpha$, Pavlov, Sanwal \& Zavlin 2006).

Here, with no information from the radio phase we must allow the $\phi$ to be freely fit. The results are interesting. For all $B$ field structures, and in particular the PFF structure, the OG model only fits satisfactorily in a small region at small $\alpha$, large $\zeta$. This means that one is viewing a nearly aligned rotator from near the spin equator, i.e. very far from the magnetic axis. This is in good agreement with the expectations of (Romani and Yadigaroglu 1995) and guarantees that the pulsar should be radio faint. In figure 11 we show surfaces computed for $w=0.18$ and three trial $\epsilon$ values. TPC shows best solutions over a range of angles and $\epsilon$. However, these have

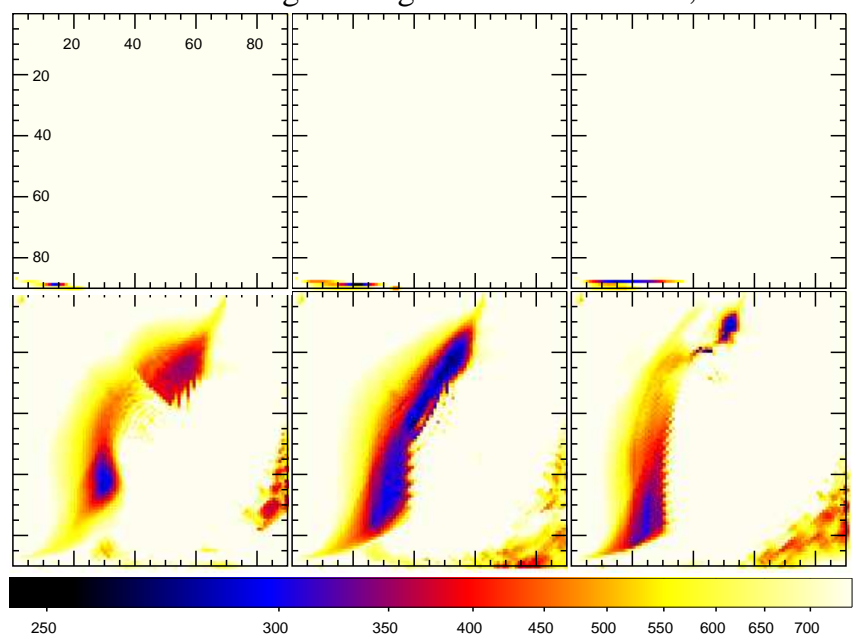

FIG. 11.- Goodness of fit surfaces for PSR J0633+1746 (Geminga), panels as for Figure 7 No quantitative external angle constraints are available, although the absence of radio emission suggests that $\beta=\zeta-\alpha>15^{\circ}$. TPC shows a number of acceptable solutions; OG has few (albeit better) solutions at small $\alpha$, large $\zeta$. 
$\Delta \chi>17$ larger than the best OG solution and show only a single peak with bridge emission (Figure 12). Thus while only few OG models are suitable, the light curve shape fits better and the viewing angles for Geminga are very well constrained. It would be very interesting to measure $\zeta$ and/or $\alpha$ from data at other wavelengths.

In Table 1, we collect these model fit results, giving the values for the externally constrained fits for the unperturbed $\epsilon=0$ field geometry for both models. In addition we quote best fits close to the RVM angles and the best global fits, when appropriate. For Geminga, with no strong radio constraint we only list the global best fits for each model.

\subsection{Luminosities}

Now that we have estimated the corrections to the all-sky flux $f_{\Omega}$, it is worth checking how well the pulsars agree with the simple luminosity law Equation (1). A major challenge to this check is the distance imprecision; of the five pulsars considered here only Vela and Geminga have parallax distance estimates. The other distances (derived from Dispersion Measure DM) are subject to appreciable uncertainty.

Geminga is in reasonable agreement with the rule only if it lies at the upper end of the present distance range. PSR $\mathrm{J} 1057-5226$ is nominally somewhat more luminous than expected, but can be accommodated if the DM distance is a slight overestimate. PSR J1709-4429, on the other hand, is much brighter than expected unless the true distance is $\sim 0.7 \mathrm{kpc}$ or the flux correction factor is as small as $f_{\Omega} \sim$ 0.07 (or some combination of the two factors). Such changes would be quite surprising. It would be very interesting to obtain a parallax distance estimate for PSR J1709-4429 to eliminate that source of uncertainty.

\section{CONCLUSIONS AND FUTURE PROSPECTS}

In (Watters et al 2009) we showed that patterns in the separation of radio and $\gamma$-ray pulse components could be used to find acceptable geometrical parameters in dipole magne-

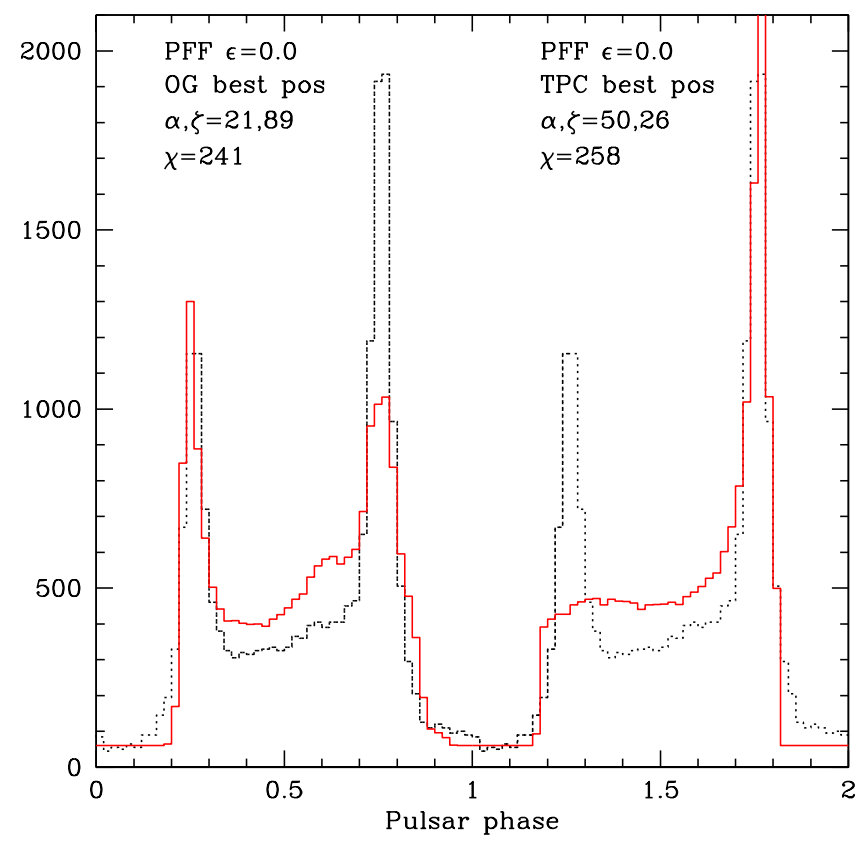

FIG. 12.- Light curves for two PFF models for PSR J0633+1746 (Geminga). The left model is for the best OG solution, the right model for the best TPC solution away from $\beta \approx 0$.

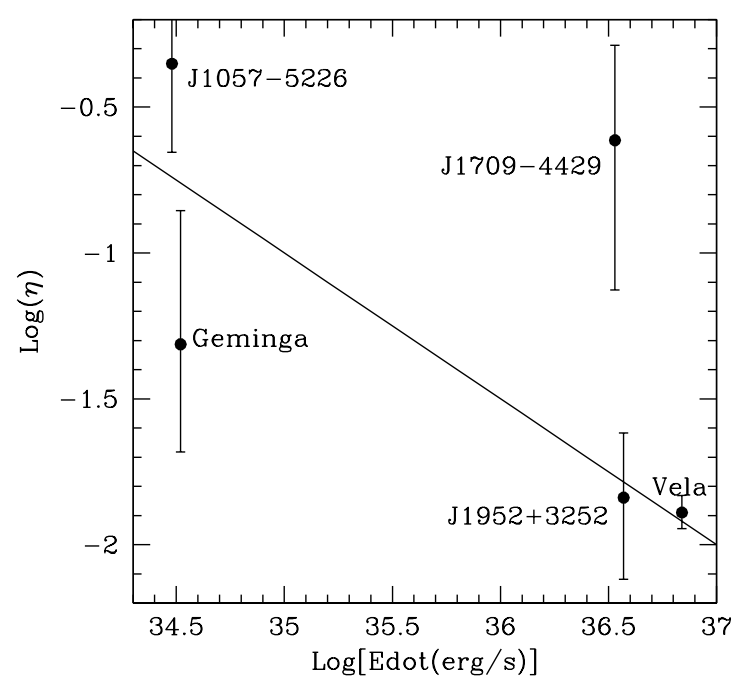

FIG. 13.- Pulsar $\gamma$-ray luminosity, corrected from the observed phaseaveraged flux to all-sky vs. the spindown luminosity. $\mathrm{OG} / \mathrm{PFF} / \epsilon=0$ points.

tosphere models for pulsar emission. Here we show that with high quality LAT light curves and reference to multiwavelength information on the inclination and viewing angles the direct comparison between the model light curve shape is even more constraining. Within the restricted context of the present computations - dipole fields, simple expressions for the gap ranges and $\gamma$-ray emissivity on the radiating surface we can already make some strong statements.

- Emission starting above the null charge surface (OG model) is strongly statistically preferred over models which have substantial emission starting from the stellar surface (eg. the TPC model). For PSR J1952+3252 the fit for the TPC model is not much worse than that for OG, but for none of the pulsars modeled here is it better, if external angle constraints are used.

- More realistic dipole field geometries, including sweep back and fields enforcing co-rotation produce improved matches to the pulsar light curves when one focuses on solutions near the orientation angles $\alpha$ and $\zeta$ implied by lower energy data. Some additional improvement can be found by using test models for field-induced current perturbations, but these are evidently not yet sufficiently realistic for detailed fits.

- The correction from observed flux to pulsar luminosity can be substantial. With these corrections a simple constant $\Phi, L_{\gamma} \propto \dot{E}(\propto$ current) law provides an improved description of the data. Nevertheless, the large departure for PSR J1709-4429 suggests additional factors are needed to understand pulsar luminosities.

- The best-fit global models are often several degrees off of the externally known $\zeta$ and $\alpha$. This difference is statistically significant and implies that the LAT data have the power to reveal perturbations to the simplified field geometry.

The robust success of the pulse profile computations described here for angles near those available from other data make it likely that the true magnetospheric geometry is fairly close to our simplified PFF model. It must be re-emphasized that this PFF model is not a complete physical description and indeed the whole electrodynamic picture of 'Hollowaytype' outer gaps is still of questionable validity. How- 
TABLE 1

PFF Model ANGLES AND EFFICIENCIES

\begin{tabular}{|c|c|c|c|c|c|c|c|c|c|c|c|c|c|}
\hline Name & $\begin{array}{l}\log (\dot{E}) \\
\operatorname{erg~s}^{-1}\end{array}$ & $w$ & $d^{\mathrm{a}}$ & $\alpha / \zeta$ & $\begin{array}{c}\alpha / \zeta^{\prime} \\
\text { External } \longrightarrow\end{array}$ & $\begin{array}{c}\alpha / \zeta / \epsilon \\
\longleftarrow\end{array}$ & $\begin{array}{c}/ \delta \phi \\
\text { TPC }\end{array}$ & $f_{\Omega}$ & $\alpha / \zeta / \epsilon$ & $\mathrm{OG}^{\chi / \delta \phi} \longrightarrow$ & $f_{\Omega}$ & $\overline{F_{>0.1 \mathrm{GeV}^{\mathrm{a}}}}$ & $\eta_{O G}$ \\
\hline Vela & 36.84 & 0.012 & $0.287_{-0.017}^{+0.019}$ & $\begin{array}{l}72 / 64 \\
\text { best } \\
\text { best }\end{array}$ & $\begin{array}{c}122 / 116[58 / 64] \\
\text { in reg } \\
\text { global }\end{array}$ & $72 / 64 / 0$ & $201 /+0.02$ & 1.06 & $\begin{array}{l}72 / 64 / 0 \\
71 / 64 /+0.2 \\
82 / 71 /-0.2\end{array}$ & $\begin{array}{r}120 /-0.03 \\
88 /-0.02 \\
34 /-0.03\end{array}$ & $\begin{array}{l}1.03 \\
0.98 \\
0.86\end{array}$ & $879.4 \pm 5.4$ & 0.013 \\
\hline $\mathrm{J} 1952+3252$ & 36.57 & 0.016 & $2.0 \pm 0.5$ & $\begin{array}{c}\mathrm{b} \\
\text { best }\end{array}$ & in reg & $\begin{array}{l}71 / 84 / 0 \\
86 / 66 /-0.2\end{array}$ & $\begin{array}{l}19 /+0.05 \\
18 /+0.05\end{array}$ & $\begin{array}{l}1.10 \\
1.10\end{array}$ & $\begin{array}{l}66 / 78 / 0 \\
71 / 71 /-0.2\end{array}$ & $\begin{array}{l}14 /+0.05 \\
14 /+0.05\end{array}$ & $\begin{array}{l}0.84 \\
0.70\end{array}$ & $13.4 \pm 0.9$ & 0.015 \\
\hline J1709-4429 & 36.53 & 0.017 & $1.4-3.6$ & $\begin{array}{c}34 / 53 \\
\text { best }\end{array}$ & $\begin{array}{c}\text { 108/127[72/53] } \\
\text { global }\end{array}$ & $31 / 49 / 0$ & $147 /+0.10$ & 1.30 & $\begin{array}{l}36 / 56 / 0 \\
47 / 53 /+0.2\end{array}$ & $\begin{array}{l}30 /-0.04 \\
19 /-0.01\end{array}$ & $\begin{array}{l}0.89 \\
0.76\end{array}$ & $124 \pm 2.6$ & 0.24 \\
\hline Geminga & 34.52 & 0.175 & $0.25_{-0.06}^{+0.12}$ & - & - & $50 / 26 / 0$ & $258 /+0.41$ & 0.93 & $21 / 89 / 0$ & $241 /+0.87$ & 0.13 & $338.1 \pm 3.5$ & 0.05 \\
\hline $\mathrm{J} 1057-5226$ & 34.48 & 0.183 & $0.71 \pm 0.2$ & best & $\begin{array}{c}\text { 105/111[75/69] } \\
\text { global }\end{array}$ & $\begin{array}{l}75 / 69 / 0 \\
41 / 08 /-0.2\end{array}$ & $\begin{array}{r}414 /+0.12 \\
37 /+0.03\end{array}$ & $\begin{array}{l}0.91 \\
0.38\end{array}$ & $\begin{array}{l}75 / 69 / 0 \\
7 / 87 /-0.2\end{array}$ & $\begin{array}{r}106 /+0.11 \\
5 /+0.11\end{array}$ & $\begin{array}{l}0.82 \\
0.09\end{array}$ & $27.2 \pm 1.0$ & 0.45 \\
\hline
\end{tabular}

${ }^{\mathrm{a}}$ From Abdo et al (2010), flux units $10^{-11} \mathrm{erg} \mathrm{cm}^{2} \mathrm{~s}^{-1}$

b $\mathrm{d} \Psi /\left.\mathrm{d} \phi\right|_{\max } \approx+3.5^{\circ} / \mathrm{deg}-$ Weisberg et al. (1999).

ever, other scenarios need to match the light curves quite closely to be competitive - this likely forces them into rather similar magnetosphere geometries. Comparison with other approaches, such as numerical magnetosphere simulations, should be fruitful. With more pulsar light curves and higher quality data being provided by the Fermi LAT, we should be able to use the departures from the predictions of these simple beaming laws to infer geometrical modifications (and work toward their physical origin) and to follow the evolution of the gap geometry and radiation with pulsar age. In a following paper we describe the additional constraints on $w(\dot{E}), \delta w$, the radial extent of the emission zone, and the pulsar evolution with age that can be extracted from the full LAT pulsar data set. This should set the context for true radiation models, which can take the next step in revealing the underlying magnetospheric physics.

We thank Jon Arons, Sasha Tchekhovskoy and Anatoly Spitkovsky for useful discussions about the field geometry. This work was supported in part by NASA grants NAS500147 and NNX10AD11G.

\section{REFERENCES}

Abdo, A.A. et al 2010, ApJ, in press.

Abdo, A.A. et al 2009b, ApJ, 696, 1084

Arons, J. 2006, 26th IAU, Parugue, JD02 \#39, 2

Bai, X.-N. \& Spitkosky, A. 2009a, ApJ submitted. arXiv0910.5740

Bai, X.-N. \& Spitkosky, A. 2009b, ApJ submitted. arXiv0910.5741

Blaskiewicz, M., Cordes J.M. \& Wasserman I. 1991, ApJ, 370, 643

Cheng, K.S., Ho, C. \& Ruderman, M. 1986, ApJ, 300, 522

Chiang, J. \& Romani, R.W. 1994, ApJ, 436, 754

Contopolous, I., Kazanas, D. \& Fendt, C. 1999, ApJ, 511, 351

Daugherty, J.K \& Harding, Alice K. 1996, ApJ, 458, 278

Deutsch, A.J. 1955, Ann. d'Ap., 18, 1

Dyks, J. \& Rudak, B. 2003, ApJ, 598, 1201.

Dyks, J., Harding, Alice K., \& Rudak, B. 2004, ApJ, 606, 1125.

Harding, A.K. 1981, ApJ, 245, 267

Johnston, S. et al 2006, MNRAS, 369, 1916

Kaburaki, O. 1980, ApSS, 67, 3.

Karastergiou, A. \& Johnston, S. 2007, MNRAS, 380, 1678.

Keith, M. et al 2009, MNRAS, in press
Lyne, A.G. \& Manchester, R.N. 1988, MNRAS, 234, 477.

Moffet, D. \& Hankins, T. 1996, ApJ, 478, 779.

Morini, M. 1983, MNRAS, 202, 495

Muslimov, Alex G. \& Harding, Alice K. 2004, ApJ, 606, 1143.

Muslimov, Alex G. \& Harding, Alice K. 2009, ApJ, 692, 140.

Ng, C.-Y., \& Romani, R. 2008, ApJ, 673, 411.

Pavlov, G.G., Sanwal, D. \& Zavlin, V.E. 2006, ApJ, 643, 1146

Radhakrishnan, V. \& Cooke, D.J. 1969, ApL, 3, 225.

Romani, R.W. 1996, ApJ, 470, 469

Romani, R. \& Yadigaroglu, I.-A. 1995, ApJ, 438, 314.

Spitkovsky, A. 2006, ApJ, 648, L51.

Watters, K.P. et al 2009, ApJ, 695, 1289

Weisberg, J. et al 1999, ApJS, 121, 171

Weltevrede, P. \& Johnston, S. 2008, MNRAS, 387, 1755.

Weltevrede, P. \& Wright, G. 2009, MNRAS, 395, 2117. 


\section{APPENDIX}

For the convenience of other researchers we present here figures that summarize the pulse properties of the TPC and OG models for the PFF dipole geometry assumptions. These may be compared with the corresponding figures in Watters et al. (2009).

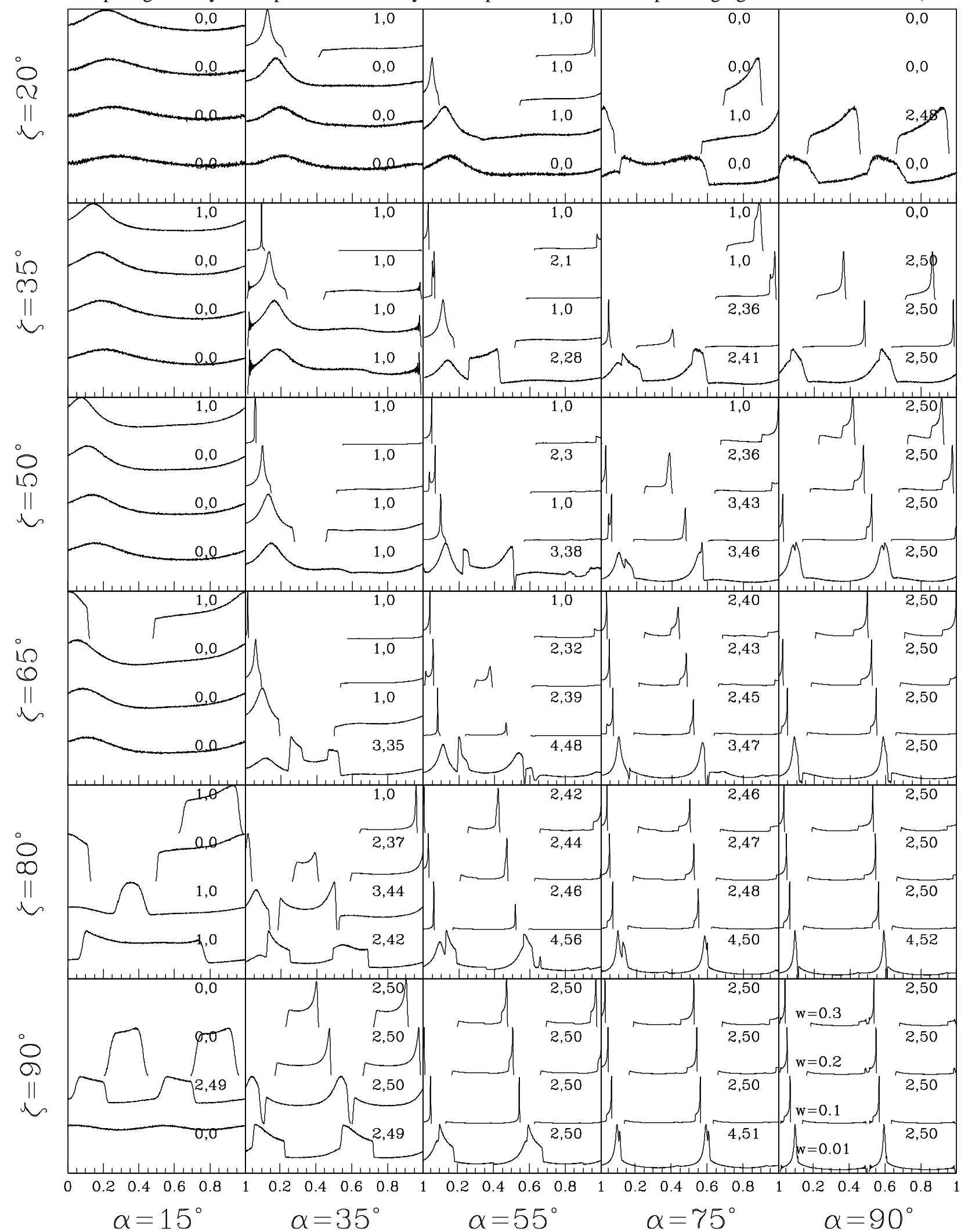

FIG. 14.- Light curves for the Two Pole Caustic (TPC) model. Each panel shows curves for four values of the gap width $w$. The curves are labeled with the number of major peaks and the peak separation, in percent. 


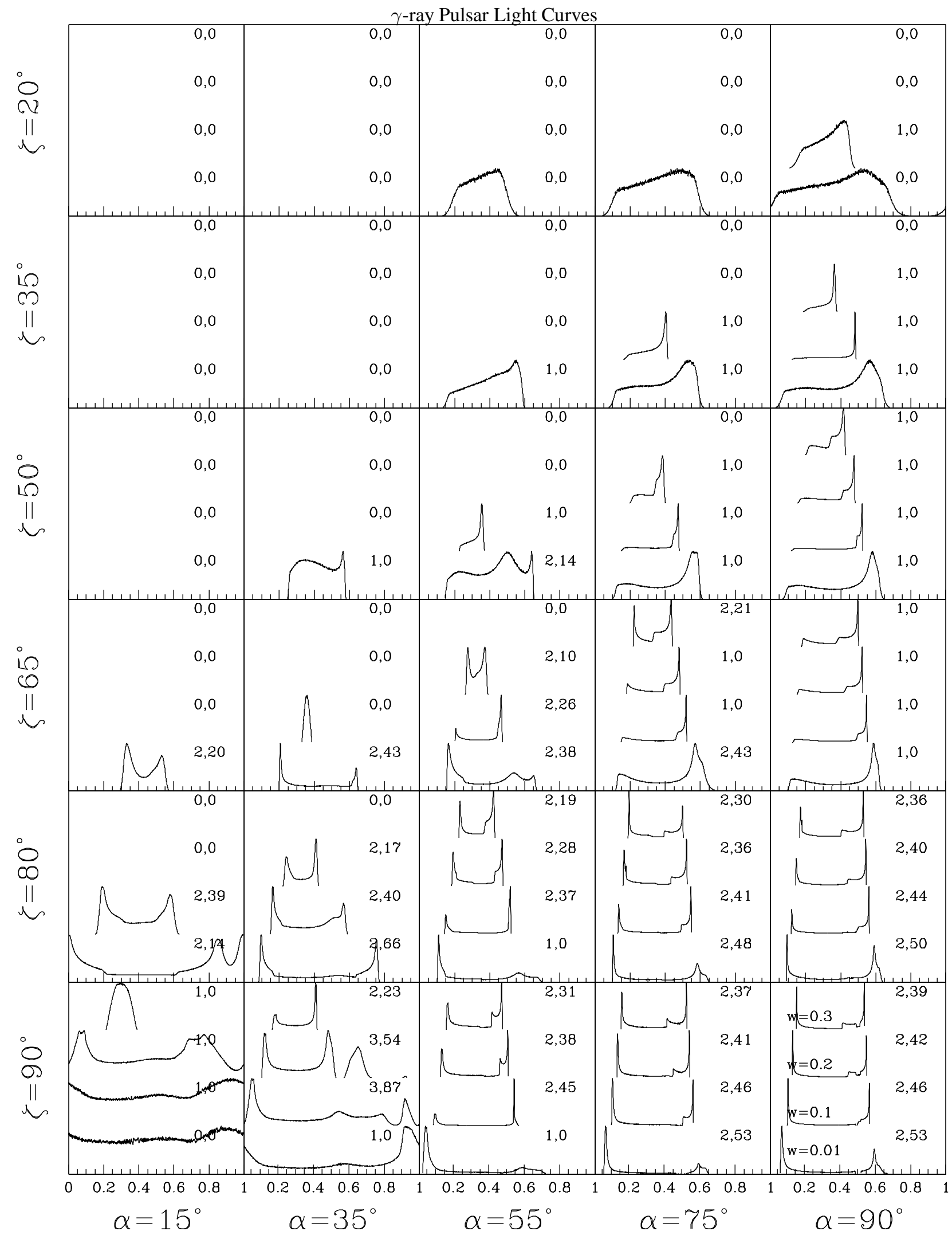




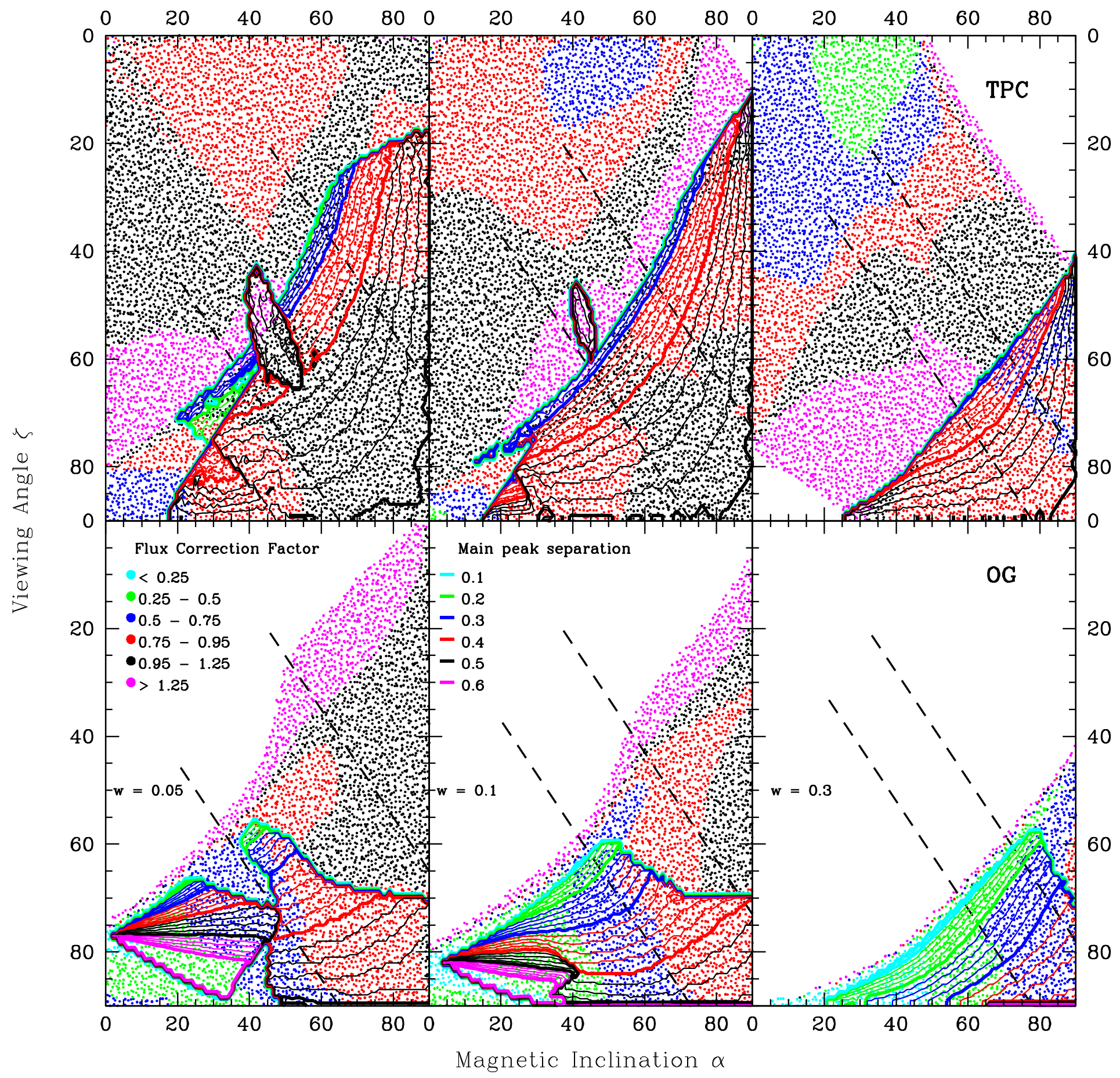

FIG. 16.- Pulse properties in the $\alpha-\zeta$ plane for two pulsar emission geometries using the PFF field. Contours show the separation $\Delta$ of the principal $\gamma$-ray pulse peaks. Bold lines mark $\Delta$ at 0.1 intervals (key bottom middle panel); fine lines mark 0.02 intervals. The background shading (key bottom left panel) gives the flux correction factor $f_{\Omega}$; large values mean that for the given $\alpha$ and $\zeta$, the pulsar displays less flux than the sky average. White regions mean little or no flux from the modeled gaps. The dashed diagonal band in each panel indicates the region where lower altitude radio pulsations are likely detected. Objects seen outside these bands tend to be Geminga-like radio-quiet pulsars. 